\title{
Analysis of expiratory tidal flow patterns as a diagnostic tool in airflow obstruction
}

\author{
M.J. Morris, R.G. Madgwick, I. Collyer, F. Denby, D.J. Lane
}

\begin{abstract}
Analysis of expiratory tidal flow patterns as a diagnostic tool in airflow obstruction. M.J. Morris, R.G. Madgwick, I. Collyer, F. Denby, D.J. Lane.@ERS Journals Ltd 1998.

ABSTRACT: An index obtained from tidal expiration, the ratio of time to peak tidal expiratory flow ( $t$ PTEF) to expiratory time $(t E)$, discriminates between groups with and without airflow obstruction in infants and children and correlates with other measurements of airflow obstruction in adults. The aim of this study was to determine whether the diagnosis of airflow obstruction could be made from an analysis of the later part of the expiratory tidal flow time curve, i.e beyond the maximum flow.

One hundred and eighteen patients attending the lung function laboratory with a putative diagnosis of airflow obstruction were studied. From uncoached tidal breathing, measurements were made of the average time constant of the respiratory system (Trs) and extrapolated volume (EV). Forced expiratory spirometry and whole-body plethysmography were performed.

In this cross-sectional study, Trs correlated with inspiratory airways resistance and forced expiratory volume in one second (FEV1), according to the linear regression equations, airway resistance $(R \mathrm{aw})=3.03$ Trs $+1.2, \mathrm{r}=0.65, \mathrm{p}<0.001$, and $\mathrm{FEV} 1 \%$ predicted $=87.8-23.7 \mathrm{Trs}, \mathrm{r}=\mathbf{0 . 5 8}, \mathrm{p}<\mathbf{0 . 0 0 1} . \mathrm{EV}$ correlated positively with overinflation, functional residual capacity $($ FRC $) \%$ pred $=152 \mathrm{EV}+103, \mathrm{r}=0.68, \mathrm{p}<0.001$.

This study shows that there is a relationship between these measurements made from analysis of tidal breathing and recognized measurements of airflow obstruction and overinflation.
\end{abstract}

Eur Respir J 1998; 12: 1113-1117.

In 1981, we made the observation that tidal flow versus time has a distinctive pattern in patients with airflow obstruction that is different from the sinusoidal appearance seen in normal subjects. To quantify this difference, we described timing indices derived from the analysis of tidal expiratory flow [1]. These indices, volume expired when the peak tidal expiratory flow reached (VPTEF)/tidal volume $(V \mathrm{~T})$ and (time to peak expiratory tidal flow $(t \mathrm{PTEF}) /$ expiratory time $(t E)$ closely correlated with each other and correlated significantly with other indices of airflow obstruction [1]. The index $t \mathrm{PTEF} / \mathrm{tE}$, has been demonstrated to be useful in predicting wheezy illness in the new-born [2], in discriminating between asthmatic and nonasthmatic children [3-5] and between healthy control subjects and children with cystic fibrosis [5] and showing response to histamine $[5,6]$, methacholine [7] and bronchodilator [3-5] in asthmatic children, though recently has not been found to be useful in the diagnosis of narrowed airways in infants [8$10]$.

In our original description of the typical flow time pattern of tidal expiration in patients with airflow obstruction, we described three parts to the curve: 1) a rapid rise to maximum flow; 2) a slow decline in flow over most of expiration, followed by; 3 ) an abrupt fall of flow to zero as the next inspiration is triggered while there is still a measurable expiratory flow. In contrast, in normal subjects, expiratory flow rises more slowly to a maximum, and then decreases gradually to zero, resulting in a smoother appearance of the tidal expiratory flow-time curve.
The index $t \mathrm{PTEF} / \mathrm{tE}$ uses only information from the first part of the expiratory flow-time curve, based on a single point. We have described two further indices $[11,12]$ in an attempt to use the information contained in the period of declining expiratory flow after the maximum flow has been reached (figs. 1 and 2). These indices are: 1) the time constant of the respiratory system (Trs); and 2) extrapolated volume (EV) (the volume of dynamic overinflation), i.e. the extra volume that would have been expired if expiration had continued to the elastic equilibrium volume.

The theoretical basis of this analysis is that if expiration is relaxed the decay of flow against time reflects the compliance and resistance of the respiratory system. OTІ et al. [13] described a simple model of the respiratory system consisting of a single compartment of constant elastance served by a pathway of constant resistance. When, in this model, expiratory flow is driven by the relaxation pressures of the lung and chest wall, there is a linear volumeflow relationship. This slope is the time constant of relaxed expiration and by analogy with an electrical model Trs is equal to resistancexcompliance of the total respiratory system (lungs+ chest wall). COMROE et al. [14] suggested that volume-flow curves of passive expiration could be used to assess the mechanical properties of the lung and chest wall.

In patients with airflow obstruction, there is evidence that inspiratory and expiratory muscles are relaxed throughout much of expiration $[11,15]$. In normal subjects, evidence points to full relaxation only occurring towards the end of expiration, inspiratory muscle activity continuing well 
a)

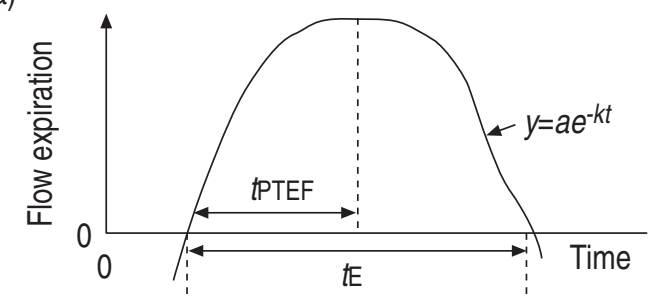

c)

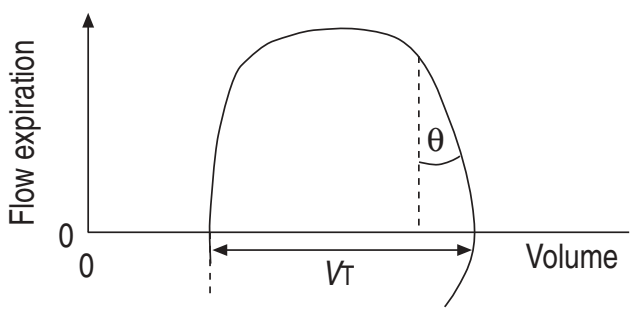

b)

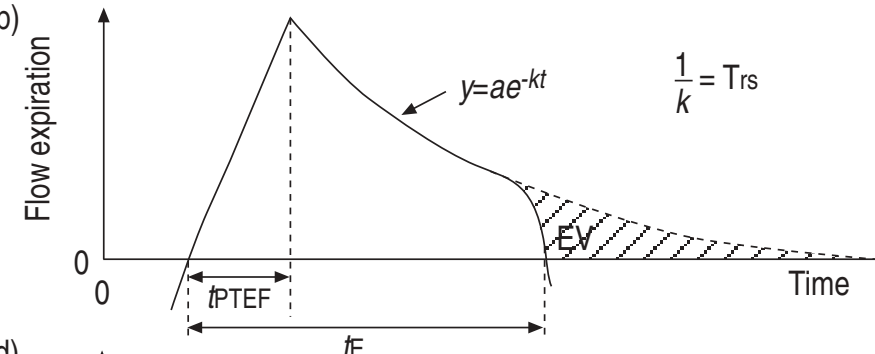

d)

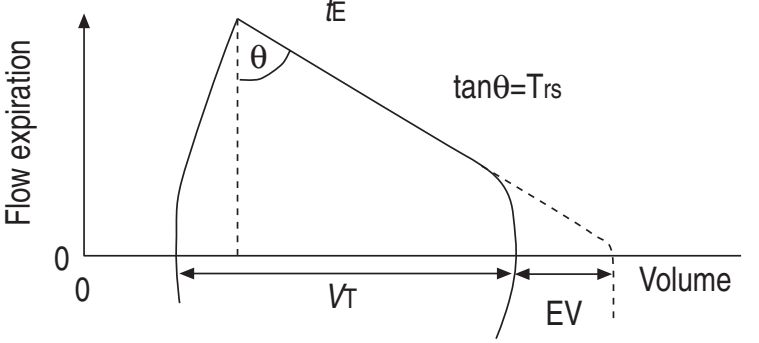

Fig 1 - Schematic representation of tidal flow-time (a,b) and flow-volume (c, d) tracings in a normal subject (a, c) and a patient with airflow obstruction $(\mathrm{b}, \mathrm{d})$. If it is assumed that flow decays exponentially against time, flow at any instant $=a e^{-k t}$ where $a$ is the flow at time zero, $t$ is time elapsed since time zero, and $k$ is the decay constant of the exponential and is the reciprocal of the time constant. $t$ PTEF: time to peak tidal expiratory flow; $t \mathrm{E}$ : expiratory time; Trs: time constant of the respiratory system; EV: extrapolated volume; $V \mathrm{~T}$ : tidal volume.

into expiration [11, 16-19]. Our hypothesis has been that if the relaxed part of tidal expiration can be identified, analysis of this portion of the flow-time or flow-volume relationships will be informative about the presence and severity of airflow obstruction.

In this study, we tested whether these two additional indices, Trs and EV, derived from uncoached tidal breathing, correlate with traditional measurements of airways resistance and overinflation, respectively.

\section{Methods}

\section{Patient selection}

One hundred and eighteen patients referred to the lung function laboratory with a putative diagnosis of airflow obstruction were tested by one of three operators (M.J. Morris, R.G. Madgwick, F. Denby). Final diagnoses included asthma in remission, chronic asthma, chronic obstructive lung disease, and bronchiectasis including cystic fibrosis, and some patients were found to have normal lung function. In 22 patients, the measurements of tidal breathing were made after the final dose of histamine in a bronchial reactivity challenge test [12]. No patients with restrictive lung disease, forced expiratory volume in one second (FEV1)/ forced vital capacity (FVC) $>80 \%$ and reduced total lung capacity (TLC), were included in this study. Sixty-two patients were male, and the age range was $7-85$ yrs $(4<18$ yrs of age), mean \pm SD $50.3 \pm 18.5, \mathrm{FEV} 1 \%$ predicted range 15-124, mean $61 \pm 28$, functional residual capacity (FRC) $\%$ pred range $67-289$, mean $140 \pm 44$, Trs range $0.23-4.26$, mean $1.39 \pm 0.70 \mathrm{~s}$, airways resistance $(R$ aw) range $0.4-20$, mean $4.7 \pm 3.3 \mathrm{cmH}_{2} \mathrm{O} \cdot \mathrm{L}^{-1} \cdot \mathrm{s}^{-1}$. The patients exhibited the full spectrum of airways narrowing from normal to very severe airflow obstruction (figs. 3-5).

\section{Equipment}

Expiratory spirometry was performed on a Vitalograph spirometer (Vitalograph, Buckingham, UK) and/or in a Jaeger body plethysmograph (Masterlab; Jaeger, Market Har- borough, Leics, UK). Static lung volumes were then measured in the constant volume body plethysmograph after computerized calibration of pressure transducers and manual calibration of the pneumotachograph with a 2-L syringe. Inspiratory Raw from 0 to $0.5 \mathrm{~L} \cdot \mathrm{s}^{-1}$ at FRC was measured in 112 patients in the Jaeger Masterlab whole-body constant-volume plethysmograph. FRC was measured with the hands compressing the cheeks while panting at a frequency of not more than $1 \mathrm{~Hz}$. The analogue flow-time tracing from a series of 10 breaths of tidal breathing (noseclip and flange mouthpiece) was taken into an Elonex PC433 via Analogue Devices 12 bit A/D interface card, with a sampling rate of $80 \cdot \mathrm{s}^{-1}$. The flow was measured either with a separate Fleisch No. 2 pneumotachometer calibrated with a known volume signal of 1 or $2 \mathrm{~L}$, or with the alreadycalibrated plethysmograph pneumotachograph. These data were analysed by M.J. Morris using the Rasp Physiologic computer program (Newbury, Berks, UK) [20], tidal breathing module (Version 3), as indicated in figs. 1 and 2. The first 10 breaths were analysed breath-by-breath and a mean value for each subject obtained for Trs and EV.

The within-subject variation for these indices has been shown to be greater in normal subjects than in patients with airflow obstruction [12]. Volume drift was corrected within the analysis program.

\section{Determination of tidal breathing indices}

In the model by OTIs et al. [13], there is an almost instantaneous rise to maximum flow, and, thereafter, the expiratory flow-volume relationship is linear if the muscles are fully relaxed and flow is being driven by the recoil pressures of the respiratory system [21, 22]. In eight normal subjects, it has been shown that such relaxation occurs during the last $20 \% \mathrm{tE}$ (mean), ranging between the last $55 \%$ and the last $4 \%$ of $t \mathrm{E}$ [16].

Trs. The slope, volume/flow, of the linear portion of the relaxed expiration has been shown to be the Trs and is equal to the total respiratory resistance multiplied by the total respiratory compliance $[13,14]$. The Rasp computer program shows the linear regression line fitted to progressively 

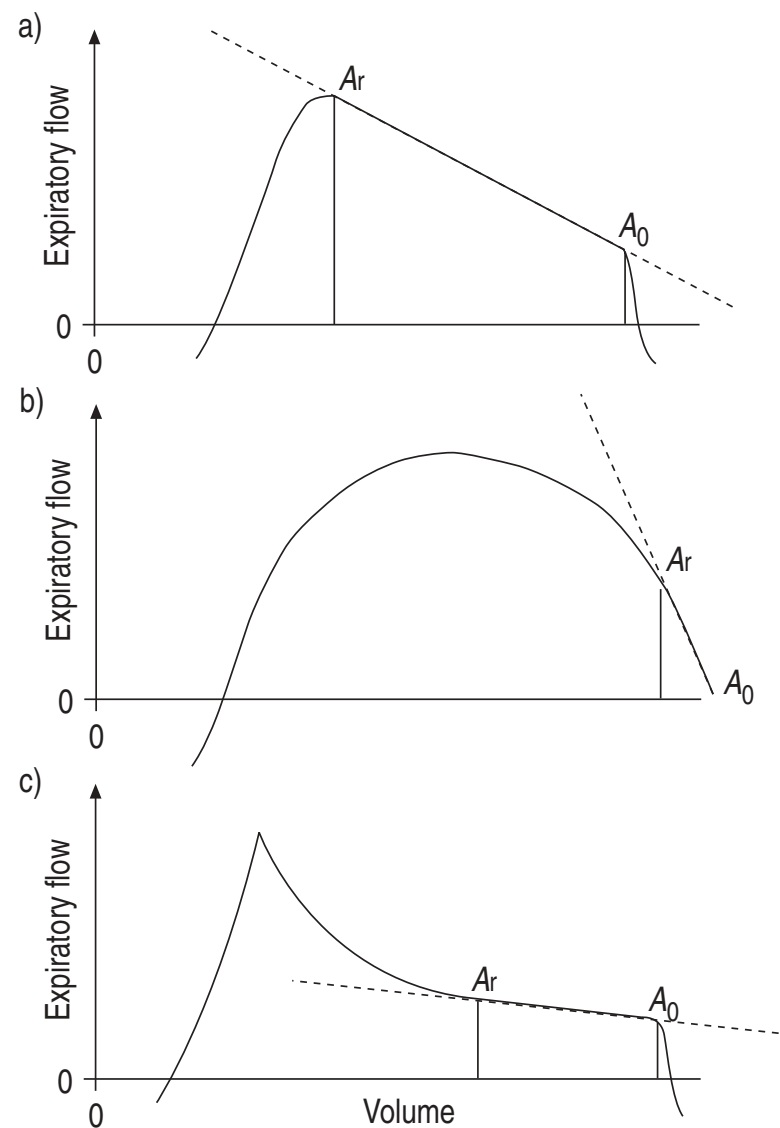

Fig. 2. - Schematic representation of the three patterns seen (see text) when a regression line was fitted to the tidal expiratory flow-volume curve. The curve was followed backwards from $A_{0}$, just before the end of expiration, to $A \mathrm{r}$, which is the point where the curve diverges from the fitted regression line.

longer portions of any curve. Using this facility, we fitted in each breath a straight line going backwards from $A_{0}$, the flow just before expiration ends (fig. 2) towards the peak until the tracing of the flow volume relationship diverged from the extrapolated retrograde portion of the fitted line. Three patterns emerged (fig. 2): 1) A single slope described the curve from $A_{0}$ back to maximum flow. 2) In sinusoidal patterns, a short distance back from $A_{0}$ the tracing came to lie beneath the extrapolated portion of the fitted line. The point at which divergence occurred was labelled $A$ r. 3) In patients with severe airflow obstruction, at $A r$ the tracing diverged to lie above the extrapolated portion of the line fitted retrogradely to the later portion of the flow-volume curve beginning at $A_{0}$.

Ar was determined by eye by a trial-and-error method noting the effect on the fitted slope of extending in small increments the length of the curve to which it was fitted. The time constant, Trs, is the gradient, volume/flow, of the regression line fitted to the tracing between $A_{0}$ and $A$ r. The assumption that is made is that between $A \mathrm{r}$ and $A_{0}$ expiration is passive. The $r^{2}$ value of the fitted regression line was used to assess the goodness of fit (i.e. linearity), an $r^{2}$ of $>0.85$ was required.

$E V$. This is the intercept on the abscissa of the flow-volume curve when the fitted regression line between $A r$ and $A_{0}$ is extrapolated to the abscissa, i.e. to zero flow (fig. 1). This is the volume that would have been expired if the

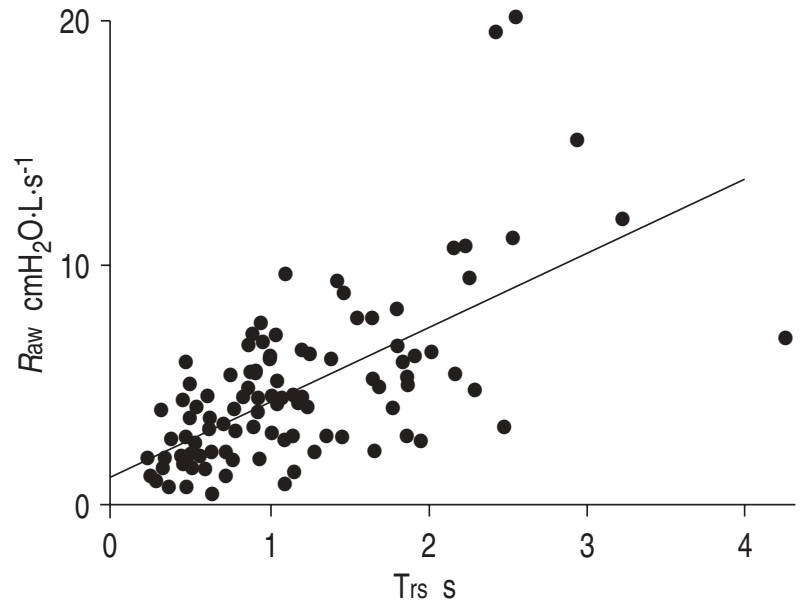

Fig. 3. - Relationship between time constant of the respiratory system $\left(T_{\mathrm{rs}}\right)$ and inspiratory airways resistance (Raw) in 112 patients. $\mathrm{r}=0.65, \mathrm{p}<0.001$.

relaxed expiration had been allowed to continue rather than being interrupted by the next inspiration. This volume is the product of $A_{0}$ and Trs.

\section{Statistics}

The mean intrasubject coefficient of variation $(\mathrm{CV})$ for Trs measured in 10 breaths was $19.7 \%$. For those subjects with an EV of Š $0.2 \mathrm{~L}$, the mean CV was $27.4 \%$. When EV was close to or equal to zero, the $\mathrm{CV}$ was greater.

The relationships of the timing index, Trs, with the established measurement of airflow obstruction, Raw, and of the new index of overinflation, EV, with an increase in FRC, were analysed by linear regression. A value of $0.8 \mathrm{~s}$ for Trs was taken as the cut-off between a normal and an abnormal test based on a value of $>2 \mathrm{cmH}_{2} \mathrm{O} \cdot \mathrm{L}^{-1} \cdot \mathrm{s}^{-1}$ for $R$ aw, as receiver operating characteristic (ROC) curve analysis showed this to be the cut-off that correctly assessed most subjects and maximized the sum of sensitivity and specificity [23].

\section{Results}

There was a strong positive correlation for a linear regression between $T_{\mathrm{rs}}$ and $R \mathrm{aw}$, slope 3.03, intercept 1.2,

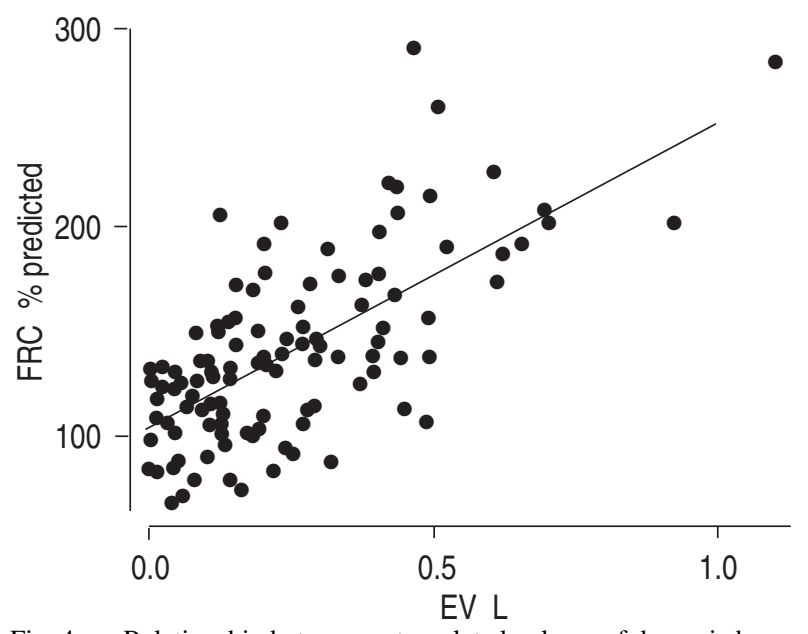

Fig. 4. - Relationship between extrapolated volume of dynamic hyperinflation (EV) and functional residual capacity (FRC) in 112 patients. $\mathrm{r}=0.68, \mathrm{p}<0.001$. 

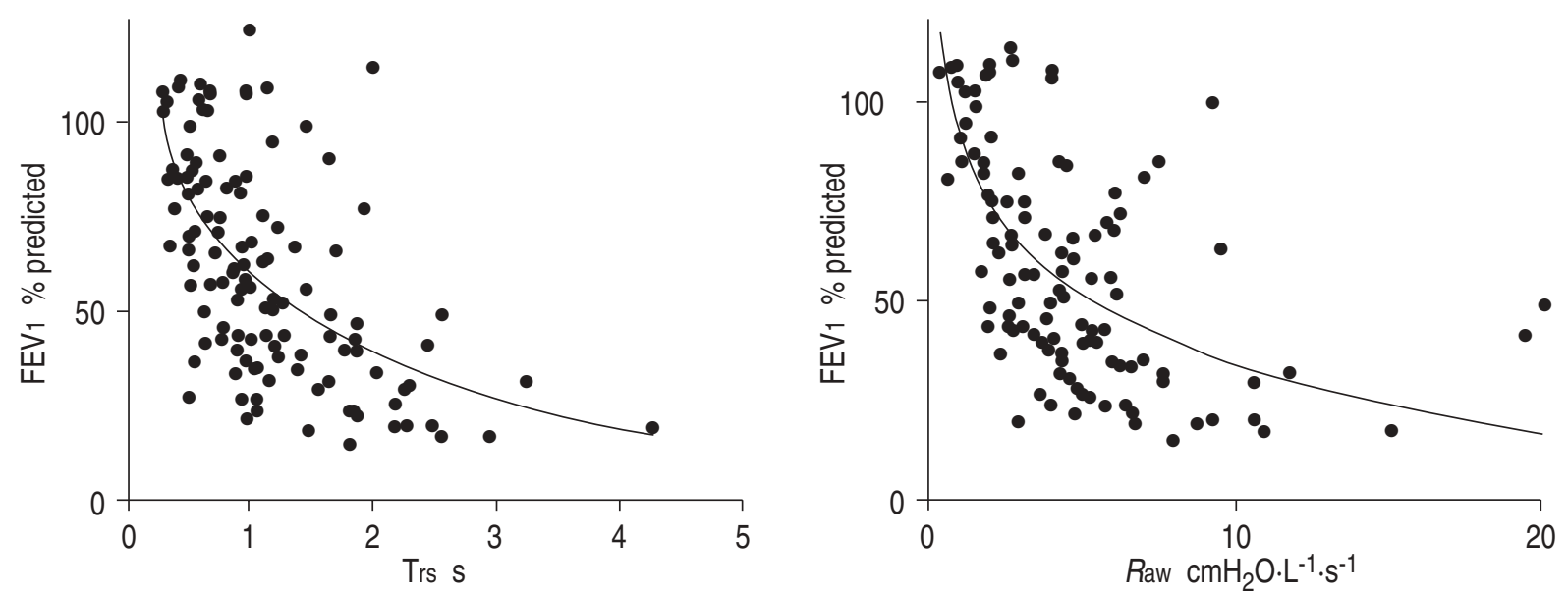

Fig. 5. - Graphs showing the similarity of the relationship between time constant of the respiratory system (Trs) and forced expiratory volume in one second $\left(\mathrm{FEV}_{1} ; \mathrm{n}=118\right)$ and inspiratory airways resistance $(R \mathrm{aw})$ and $\mathrm{FEV}_{1}(\mathrm{n}=112)$. In both, the relationship is better described by a logarithmic regression than by a linear regression. Raw versus FEV1 \% predicted linear regression, FEV1 \% pred=79.5-4.3 Raw, $\mathrm{r}=0.51$, $\mathrm{p}<0.001$, logarithmic regression $\mathrm{r}=0.64, \mathrm{p}<0.001$. Trs versus FEV1 \% pred linear regression, FEV1 \% pred=87.8-23.7 Trs, $\mathrm{r}=0.58, \mathrm{p}<0.001$, logarithmic regression $\mathrm{r}=0.62$, $\mathrm{p}<0.001$.

$\mathrm{r}=0.65$, p $<0.001$ (fig. 3), and between EV and FRC\% pred, slope 152, intercept 103, r=0.68, p<0.001 (fig. 4). A logarithmic regression described the relationship between Trs and FEV 1 better than did a linear regression, $r=0.62$ versus 0.58 (fig. 5). The relationship of Trs with Raw was closer than that with $\mathrm{FEV}_{1} \%$ pred, $\mathrm{r}=0.65$ versus $\mathrm{r}=0.58$ (figs. 3 and 5). For comparison, the relationship between a traditional tidal breathing measurement of airways obstruction, Raw, and FEV1, an accepted index derived from a maximally forced expiration is shown (fig. 5). This relationship was essentially no closer than the relationship between Trs, also measured during tidal breathing, and FEV1 $\left(\mathrm{r}_{R \mathrm{Rw}}=\right.$ 0.51 versus $r_{T r s}=0.58$, linear regression; $r_{\text {Raw }}=0.64$ versus $\mathrm{r}_{\mathrm{Trs}}=0.62$, logarithmic regression).

The sensitivity of a value for Trs of $>0.8$ s for predicting an abnormal value of $R$ aw $\left(>2 \mathrm{cmH}_{2} \mathrm{O} \cdot \mathrm{L}^{-1} \cdot \mathrm{s}^{-1}\right)$ was $75 \%$, and the specificity $85 \%$.

The mean $( \pm \mathrm{SD})$ values of Trs $0.54 \pm 0.26 \mathrm{~s}, t \mathrm{PTEF} / \mathrm{tE}, 34.7 \pm$ $10.6 \%$, and EV, $0.08 \pm 0.06 \mathrm{~L}$, in normal subjects (Raw $<2$ $\left.\mathrm{cmH}_{2} \mathrm{O} \cdot \mathrm{L}^{-1} \cdot \mathrm{s}^{-1}\right)$ and in those with airflow obstruction, $1.28 \pm$ $0.71 \mathrm{~s}, 23.0 \pm 10.4 \%$ and $0.29 \pm 0.20 \mathrm{~L}$, were significantly different (t-test, $\mathrm{p}<0.001$ for all three indices).

\section{Disscussion}

This cross-sectional study has shown that the indices, Trs and EV, derived from an analysis of tidal expiration correlate with recognized indices of airflow obstruction and of overinflation. This is a pragmatic study, with the data having been obtained in a routine service laboratory after the usual calibration procedures. Patients were given the usual coaching and instructions for the standard measurements, and the tests were carried out by one of three technicians. Although all subjects did not perform the standard manoeuvres perfectly, they are all included in this analysis as their measurements were deemed acceptable for reporting to the referring physician. The benefit of the tidal breathing test is that the subjects are not asked to breathe in any special way. These results are presented in the belief that they indicate how well these new tests will perform in a laboratory carrying out routine pulmonary function testing.
In any assessment of a new method of measuring directly or indirectly pulmonary resistance, the difficulty of the absence of an absolute method with which to compare becomes apparent. FEV $1 \%$ pred is a robust, reliable measurement, but it is made after a maximum inspiration that alters the compliance characteristics of the lung and airways. It depends on cooperation, co-ordination, maximum effort, and an understanding of what is required, on the part of the patient. During this maximally forced manoeuvre, there is dynamic compression of large airways, so it is not surprising that measurements of airway calibre made during tidal breathing do not correlate perfectly with the flow measurements made during a maximally forced expiration from TLC. This is also true for the correlation of the recognized measure Raw with FEV1. Raw is measured during tidal breathing and does not correlate much better with FEV1 than does Trs in these patients (fig. 5). Additional difficulty arises from there being a wide range of predicted values for FEV1 in normal subjects. A subject with measured FEV1 at the lower end of the normal range $(S D=0.5 \mathrm{~L})$ may be normal or may have airflow obstruction that has reduced the FEV1 by as much as $2 \mathrm{~L}$. The standard deviation for FEV1 is the same for all values of FEV1, so the percentage predicted becomes less useful as a measure of abnormality as predicted FEV1 decreases, e.g. $60 \%$ pred may be normal for a small elderly female while clearly signalling abnormality in a young tall male. Thus, although FEV1 expressed as a percentage of that predicted in general detects abnormality, there will be cases when the inference of normality, or otherwise, from this measurement will be erroneous. Similarly, plethysmographic measurements of tidal airways resistance and of overinflation may be inaccurate due to the pressure measured at the mouth underestimating alveolar pressure [24, 25] or may differ from tidal resistance measured without panting. It can be seen that imperfect correlations between the new tidal breathing indices and standard measurements obtained from maximally forced expiratory manoeuvres and from plethysmography may occur because of inaccuracies in the standard measurements as well as in the new indices.

This index, Trs, is directly related to the mechanical characteristics of the relaxed respiratory system. Unlike Raw, Trs includes tissue resistance of the lung and chest wall, 
which, however, becomes less important as the severity of airflow obstruction increases. We have endeavoured to identify the portion of the expiratory tidal flow-time and flow-volume curves during which expiration is passive. We appreciate that a single exponential does not fully describe passive expiration in normal subjects [26]. However, in this study, in the normal subjects, the volume over which the time constant is measured is so small that this approximation becomes acceptable. The fit of a single exponential was good, $\mathrm{r}^{2}>0.85$. In severe airflow obstruction, the time constant becomes increasingly long as expiration proceeds, expired gas coming progressively from more obstructed regions of the lung. We have chosen the longest time constant at the end of tidal expiration (fig. 2). As well as the total respiratory resistance and the distribution of resistances along parallel pathways, the compliance of the total respiratory system will influence the time constant $\left(\mathrm{T}_{\mathrm{rs}}=\right.$ respiratory system resistance $\times$ respiratory system compliance). The increase in static lung compliance that occurs in emphysema will lengthen Trs, but this effect will be counteracted by the frequency-dependent decrease in dynamic compliance that occurs in airflow obstruction.

EV represents the overinflation above its relaxation volume of the part of the lungs participating in tidal ventilation. Therefore, it does not include the increase in the actual relaxation volume seen in emphysema due to a loss of lung recoil and in long-standing airflow obstruction due to a loss of chest wall recoil $[11,27]$. Nor does it include the volume of gas trapped behind airways closed in the tidal range. The reasonably good correlation of EV with FRC \% predicted suggests that these various causes of overinflation move in parallel with each other as airflow obstruction increases.

This analysis of tidal breathing will be useful in patients in whom maximally forced manoeuvres are unreliable, unobtainable or cause distress. Although there are some data on the variability of these indices [12], further work being undertaken includes studying a large broadly based group of normal subjects. The subjective nature of the method of determining the point where the curve diverges from the fitted regression line, i.e. which part of the expiration is passive, is a disadvantage of this physiologically based analysis. The accompanying paper describes a further analysis of tidal breathing in which preset criteria are used, and the analysis is entirely objective.

Acknowledgements: We are grateful to P. McShane for his help with the statistical analysis of the data and to A. Thomson for her critical reading of the manuscript and helpful suggestions.

\section{Reference}

1. Morris MJ, Lane DJ. Tidal expiratory flow patterns in airflow obstruction. Thorax 1981; 36: 135-142.

2. Martinez FD, Morgan WJ, Wright AL , Holberg CJ, Taussig LM. Diminished lung function as a predisposing factor for wheezing respiratory illness in infants. $N$ Engl $J$ Med 1988; 319: 1112-1117.

3. Carlsen KH, Lodrup Carlsen KC. Tidal breathing analysis in awake young children with and without asthma, and their response to salbutamol. Eur Respir J 1994; 7: 2154-2159.

4. Lodrup Carlsen KC, Halvorsen R, Ahlstedt S, Carlsen KH. Eosinophil cationic protein and tidal volume loops in children aged 0-2 yrs of age. Eur Respir J 1995; 8: 1148-1154.

5. Van der Ent CK, Brackel HJL, Van der Laag J, et al. Tidal breathing analysis as a measure of airway obstruction in children three yrs of age and older. Am J Respir Crit Care Med 1996; 153: 1253-1258.

6. Cutrera R, Filtchev SI, Merolla R, Willim G, Haluska J, Ronchetti R. Analysis of expiratory pattern for monitoring bronchial obstruction in school-age children. Pediatr Pulmonol 1991; 10: 6-10.

7. Benoist MR, Brouard JJ, Rufin P, Delacourt C, Waernessyckle $S$, Scheinmann P. Ability of new lung function tests to assess methacholine-induced airway obstruction in infants. Pediatr Pulmonol 1994; 18: 308-316.

8. Aston H, Clarke J, Silverman M. Are tidal breathing indices useful in infant bronchial challenge tests? Pediatr Pulmonol 1994; 17: 225-230.

9. Clarke JR, Aston H, Silverman M. Evaluation of a tidal expiratory flow index in healthy and diseased infants Pediatr Pulmonol 1994; 17: 285-290.

10. Seddon PC, Davis GM, Coates AL, et al. Do tidal expiratory flow patterns reflect lung mechanics in infants? Am J Respir Crit Care Med 1996; 153: 1248-1252.

11. Morris MJ, Madgwick RG, Frew AJ, Lane DJ. Breathing muscle activity during expiration in patients with chronic airflow obstruction. Eur Respir J 1990; 3: 901-909.

12. Morris MJ, Madgwick RG, Lane DJ. Analysis of tidal expiratory flow pattern in the assessment of histamineinduced bronchoconstriction. Thorax 1995; 50: 346-352.

13. Otis AB, Fenn WO, Rahn H. Mechanics of breathing in man. J Appl Physiol 1950; 2: 592-607.

14. Comroe JH, Nisell OT, Nimms RG. A simple method for concurrent measurement of compliance and resistance to breathing in anaesthetized animals and man. J Appl Physiol 1954; 7: 225-228.

15. Citterio G, Agostoni E, del Santo A, Marazzini L. Decay of inspiratory muscle activity in chronic airflow obstruction. J Appl Physiol: Respir J Environ Exercise Physiol 1981; 51: 1388-1397.

16. Shee CD, Ploy-Sang-Sang Y, Milic-Emili J. Decay of inspiratory muscle pressure during expiration in conscious humans. J Appl Physiol 1985; 58: 1859-1865.

17. Agostoni E. Dynamics. In: Campbell EGM, Agostoni E, Newsome Davis J, eds. The Respiratory Muscles Mechanics and Neural Control. London, Lloyd-Luke (Medical Books), 1970; p. 96.

18. Petit JM, Milic-Emili J, Delhez L. Role of the diaphragm in conscious normal man: an electromyographic study. $J$ Appl Physiol 1960; 15: 1101-1106.

19. Green JH, Howell JLB. Correlation of intercostal muscle activity with respiratory airflow in conscious human subjects. J Physiol Lond 1959; 19: 471-476.

20. Cumberland RH. RASP Respiratory Analysis Program, User's Guide. Newbury, Physio Logic.

21. Pierce JA. Studies of free collapse in the intact human lung. J Lab Clin Med 1959; 54: 96-106.

22. McIlroy MB, Tierney DF, Nadel JA. A new method for measurement of compliance and resistance of lungs and thorax. J Appl Physiol 1963; 18: 424-427.

23. Altman DC. Practical Statistics for Medical Research Chapman \& Hall, 1993; pp. 417-418.

24. Shore S, Milic-Emili J, Martin JG. Assessment of body plethysmographic technique for the measurement of thoracic gas volume in asthmatics. Am Rev Respir Dis 1982; 126: 515-520

25. Stanescu DC, Rodenstein D, Cauberghs M, Van de Woestijne KP. Failure of body plethysmography in bronchial asthma. J Appl Physiol Environ Exercise Physiol 1982; 52: 939-948.

26. Chelucci GL, Brunet F, Dall' Ava-Santucci J, et al. A single compartment model cannot describe passive expiration in intubated, paralysed humans. Eur Respir J 1991; 4: 458-464.

27. Sharp JT, Van Lith P, Vey Nuchprayoon C, Brinley R, Johnson FN. The thorax in chronic obstructive lung disease. Am J Med 1968; 44: 39-46 